\title{
RECENT ADVANCES IN PATHOLOGY
}

\author{
E. VON HAAM, \\ Department of Pathology, The Ohio State University
}

Research in the general and special fields of pathology during 1945 has been handicapped by World War II, with its great demand on manpower and the numerous pressing problems it put before the scientific minds of the nations at war. For this reason we find what little progress has been achieved in the fields of pathology nearly exclusively recorded in the North and South American literature. For the purpose of a better survey, the contributions which impressed the reviewer as "advances" in the fields of pathology will be discussed under the sections of: pathology of infectious diseases, intoxications, nutritional and metabolic disorders, circulatory disturbances, and diseases of unknown origin. Advances in the pathology of tumors, endocrine and nervous disorders are being reviewed by other members of the symposium in the medical science section and will be therefore omitted.

\section{THE PATHOLOGY OF INFECTIOUS DISEASES}

One of the most interesting contributions in this field seems to the reviewer a series of articles by Cavelti and Cavelti (1) on the pathogenesis of glomerulonephritis. They were able to produce in 127 out of 208 animals truly progressive lesions of glomerulonephritis by injecting a mixture of streptococci with homologous kidney antigen. Twenty-four animals died in the stage of acute glomerulonephritis; in 27 the glomerulonephritis went into a latent stage, and in 34 animals slowly progressive chronic glomerulonephritis was observed.

Another successful attempt to reproduce a frequently observed human infection in animals is reported by Clawson (2), who observed valvular lesions in rate after the intracardiac injection of Strep. viridens and Strep. hemolyticus. The lesions were not caused by the toxic effect of the organism, nor were they caused by an allergic reaction to the organism. Microscopically the lesions resembled closely those observed in acute rheumatic valvulitis in man.

The pathognomonic significance of the Masson body in rheumatic lesions of the lung were disputed by Herbut and Manges (3). From a study of 505 cases of unselected lung lesions over a period of 15 years, the authors concluded that Masson bodies represent intra-alveolar exudate in various stages of organization and are not specific for rheumatic fever or rheumatic pnuemonitis.

In continuation of their experiments on the relationship between swine brucellosis and human Hodgkin's disease, Brown, Forbus and Kerby (4) emphasized the similarity in the reactions produced in the lymph nodes of the hog by infection with the Brucella strain isolated from a human case of Hodgkin's disease and the strain naturally occurring in swine. On the other hand, they admitted that they have been unable to produce a disease entity comparable with human Hodgkin's disease.

In the field of fungus infections we find the interesting observation of Butt and Hoffman (5), who obtained close to $26 \%$ positive coccidioidin reactions in 700 cases admitted to the Santa Fe Coast Lines Hospital in Los Angeles. Autopsy of cases with positive skin tests revealed nodular fibrous lesions in which spherules and endospores of $\mathrm{C}$. immitis were present in $45.4 \%$ of the cases with positive reactions.

The experiences of American doctors in the Pacific and African theaters of war gave rise to numerous contributions in the field of pathology in tropical diseases. As an example we may quote the study of Rifkin and Thompson (6) on the changes in early filariasis as observed in cases received from various islands in the South Pacific. The acute stage observed during the invasion of the parasite is mani- 
fested by typical allergic reactions which are always local but may be also systemic. The subacute stage of infection is characterized by the development of granulation tissue in the lymphatic organs harboring the parasite. The chronic stage is characterized by nonspecific fibrous overgrowth in the areas of the degenerating parasites. The latter is then followed by lymph stasis and elephantiasis.

Also from the theater of the Pacific come our experiences with scrub typhus, or Tsutsugamushi disease. Allen and Spitz (7) compared the pathology of scrub typhus with that of other ricksettsia diseases. The focal encephalitis in scrub typhus and epidemic typhus can be well differentiated from the microinfarcts of Rocky Mountain spotted fever. Only slight vascular damage is found in scrub typhus in contrast to epidemic typhus. The authors believe that the fibrinoid degeneration of collagen, the necrotic changes in lymph nodes and spleen, and the acute diffuse glomerulonephritis are evidence of the action of allergens in ricksettsia diseases.

Numerous papers deal with the pathology of virus diseases. Pinkerton, Smiley and Anderson (8) studied the cytology of infantile giant cell pneumonia. They found cytoplasmic and nuclear inclusions which seemed identical with those seen in distemper infections of dogs and lower animals. This observation suggested to them a possible etiologic relationship between both diseases.

Lucas and Riser (9) studied the intranuclear inclusions found in the panleukopenia of cats. They found two types of granular nuclear inclusion bodies: clustered granular, and diffuse granular ones. Morphologically these inclusion bodies are identical with those found in the liver of yellow fever and severe burns, but the developmental cycle of the inclusion bodies seems a different one.

Lillie and Armstrong (10) studied the pathology of lymphocytic choriomeningitis in mice. If other routes of inoculation than the cerebral one were employed, the changes in the visceral organs were more striking. Fatty degeneration and focal necrosis of the liver, polyserositis of pleura and peritoneum, focal necrosis in thymus, spleen, lymph nodes and bone marrow were present in animals injected parenterally with the virus. From this study it appears probable that the spontaneous infections not using the intracerebral route of inoculation could produce visceral lesions more frequently, and that such lesions may well dominate the picture.

Saphir (11) reported the autopsy findings of 17 patients dying from poliomyelitis. In 10 cases diffuse lymphocytic myocarditis was present, in 6 cases interstitial pneumonia was found, in 5 bronchopneumonia. The author suggested that the myocarditis, which can only be detected by microscopic examination, may be responsible for the sudden death of some patients.

\section{PATHOLOGY OF INTOXICATIONS AND TRAUMA}

The interest of the pathologist in lesions encountered in various industrial occupations is evidenced by a series of articles in the field. Haythorn and Taylor (12) attempted to throw light upon the puzzling fact that in many cases patients with a considerable chemical silica content in their lungs did not show any clinical or pathological findings of the disease. Silica was extracted from silicotic lungs under careful preservation of the physical and chemical status and injected into rabbits and guinea pigs. Identical experimental lesions were obtained as with crystalline silica. These experiments proved to the authors that silica recovered from the lung has not lost any of its toxic properties by its "sojourn" through the body tissues.

In studying the effect of beryllium intoxication, Gardner and Heslington (13) produced atrophy of the spleen, progressive cirrhosis of the liver, and cortical sclerosis of the long bones, spines, and ribs. Seven rabbits surviving the injection of zinc beryllium silicate for seven months developed malignant osteosarcomas with metatases in 4 animals. 
Hirsch and Russell (14) reported the case of a furniture manufacturer who died with chronic progressive induration of both lungs which had the microscopic picture of lipoid pneumonia. Extraction of the lungs with alcohol ether yielded a large amount of shellac-like material which when injected into rabbits caused necrosis and a marked exudative and fibroblastic inflammation of the lung.

The steady increase in the use of sulfa drugs and other antibodies has produced fatal lesions which have been studied by the pathologist. Black-Schaffer (15) summarized the changes found in 5 cases of fatal sulfonamide intoxications as follows: vascular lesions ranging from intramural edema to necrosis of vessel walls, and cellular exudates in skin, renal pelvis, mediastinum, gastro-intestinal tract, and other organs showing macrophages in the stage of active phagocytosis. The author believes that both types of lesions are identical with those found in experimental protein anaphylaxis.

Herbut and Scaricaciottoli (16) reported two fatal cases following sulfadiazine administration. They too emphasize the anaphylactic nature of the reaction, particularly of the diffuse hepatic necrosis.

The recent catastrophe in Boston and the use of fire as a potential weapon of war reawakened the interest in the pathology caused by excessive heat. Moritz, Henriques, and McLean (17) studied the effect of inhaled hot air on the respiratory tract. They found that the burning of the skin and mucosa of the mouth, together with fatal obstructive edema of the glottis preceded and prevented the appearance of thermal pneumonitis.

Baker (18) studied 96 cases in which cutaneous burns or complications thereof were the chief cause of death. The author emphasized the lack of specific histopathological findings and attributed most of the changes to shock, secondary infection, or in rare instances, hemoglobinemia.

\section{NUTRITIONAL DISTURBANCES AND METABOLIC DISORDERS}

A very interesting paper regarding the liver changes in pellagra was published by Gillman and Gillman (19). The authors performed over 600 biopsies in 120 cases of various types of nutritional deficiency. In all instances they could find evidence of liver damage ranging from minimal fatty changes to severe and progressive cirrhosis with iron pigmentation. The authors speculated upon the cause of this iron retention and came to the conclusion that it represents a sign of a disturbed mitochondrial function. They also emphasized the diagnostic importance of pigmentation with cirrhosis (pigment cirrhosis) in cases of pellagra.

The distribution of phosphatase in the liver under various metabolic conditions was studied by Wachstein'(20). He found that the atrophic liver cells of starving animals and the hypertrophic liver cells of protein deficient animals showed an increase in alkaline phosphatase. Toxins disturbing the cellular metabolism, such as phosphorus and carbon tetrachloride, produced a decrease in the enzymatic activity of the injured lever cells.

Fitzgerald and Kinney (21) reported a case of rare metabolic disorder: intestinal lipodystrophy, or Whipple's disease. The case was complicated by the presence of an acute hemolytic anemia and leukocytosis. Lindsay and Knorp (22) studied a case of primary systemic amyloidosis, a condition much rarer in the United States than on the Continent.

\section{PATHOLOGY OF CIRCULATORY DISTURBANCES}

The question of the importance of cholesterol in the production of arteriosclerosis was reopened by Pollak (23), who demonstrated that the lesions produced in the rabbit aorta are comparable to the lesions in the human. Ligation of both carotid arteries, both jugular veins or of one carotid artery and one jugular vein 
produced lesions in the brain suggestive of hypertension. Those changes differ from the atherosclerotic lesions and can be explained by the impairment of the rate of blood flow with concomitant changes in the intravascular tension.

Interesting experiments in the field of hypertension are the successful attempts of Dick (24) to produce high blood pressure in dogs by injecting intravenous doses of live streptococci from various sources. The kidneys showed the picture of benign nephrosclerosis with scarring of the surface, fibrosis of glomeruli, and atrophy of tubules.

Flory (25) found 9 cases among 267 autopsies of persons with advanced atherosclerosis of the aorta showing evidence of embolic occlusions of smaller vessels in various visceral organs (spleen, pancreas, and kidney). He could produce the same type of lesion by injecting material scraped from atheromatous ulcerations of human aortas in the pulmonary vessels of rabbits.

Holyoke (26) studied 70 hearts with the injection technic of Schlesinger. In 12 instances occlusion of branches of the coronary vessels could be found at several points. In all hearts showing narrowing of the coronary branches interarterial anastomoses could be demonstrated. The author believes that the prompt establishment of such anatomosis could minimize greatly the danger of myocardial infarction in case of coronary occlusion. It is his opinion that other factors than simple coronary occlusion must play a part in the production of myocardial scars.

The importance of proper peripheral circulation was stressed in a study on the pathology of trench foot by Friedman (27). He concluded that all injuries resulting from exposure to low temperature followed the same train of events. A disturbance in the circulatory mechanism leads to stagnation of blood in the smaller vessels with thrombosis and gangrene, which then becomes complicated by secondary infection. Permanent changes produced in these vessels by the first exposure is the cause for the delayed sensitivity to cold temperature.

\section{DISEASES OF UNKNOWN ETIOLOGY}

In the field of diseases of unknown origin we may quote two studies which although they failed to prove the etiology advanced our knowledge of the diseases.

Bevans (28) studied intensively two cases of generalized scleroderma. In addition to the typical skin lesion she found myocardial fibrosis, perivascular and peribronchial fibrosis of the lung, and extensive vascular lesions in the medium sized and smaller arteries of the kidney. There were also present an atrophy of the smooth muscle of the esophagus and patchy atrophy of the smooth muscle tissue throughout the intestinal tract. The author emphasized the vascular lesions and degenerative changes of smooth muscle tissue as important factors in the pathogenesis of the disease.

Bayley, Lindberg and Baggenstoss (29) reported the first case of Loeffler's syndrome which came to autopsy. The syndrome, described in 1932, is characterized by a transitory pulmonary lesion which is usually interpreted as pneumonia, peripheral eosinophilia, and a good prognosis. A 59 year old woman complained of severe cough, restlessness and anorexia. Her blood count showed $35 \%$ eosinophils, and the x-ray picture showed dense shadows in both upper lobes. The patient improved but recurrence of her symptoms brought her back three months later. Death occurred five days after her second admission. The histological examination of the lungs showed nodular areas of fibrosis, eosinophilis, plasma cells, lymphocytes and some giant cells. Necrosis of medium sized and small arteries and veins was found with an exudate consisting largely of eosinophils and plasma cells. No evidence of a microorganism could be found bacteriologically or histologically. 


\section{BIBLIOGRAPHY}

1. Cavelti, P. A., and Cavelti, E. S. Studies on the pathogenesis of glomerulonephritis. II. Production of glomerulonephritis in rats by means of autoantibodies to kidney. Arch. Path., 40: 158, 1945.

III. Clinical and pathologic aspects of the experimental glomerulonephritis produced in rats by means of autoantibodies to kidney. Arch. Path., 40: 163, 1945.

2. Clawson, B. J. Experimental endocarditis (rheumatic-like and bacterial) in rats. Arch. Path., 40: 153, 1945.

3. Herbut, P. A., and Manges, W. E. The "Masson body" in rheumatic pneumonia. Am. J. Path., 21: 741, 1945.

4. Brown, I. W., Forbus, W. D., and Kerby, G. P. The reaction of the reticulo-endothelial system in experimental and naturally acquired brucellosis of swine. Am. J. Path., 21: 205, 1945 .

5. Butt, E. M., and Hoffman, A. M. Healed or arrested pulmonary coccidioidomycosis. Correlation of coccidioidin skin tests with autopsy findings. Am. J. Path., 21: 485, 1945.

6. Rifkin, H., and Thompson, K. J. Structural changes in early filariasis. Arch. Path., 40: 220,1945

7. Allen, A. C., and Spitz, S. A comparative study of the pathology of scrub typhus (Tsutsugamushi disease) and other richettsial diseases. Am. J. Path., 21: 603, 1945.

8. Pinkerton, H., Smiley, W. L., and Anderson, W. A. D. Giant cell pneumonia with inclusions. A lesion common to Hecht's disease, distemper and measles. Am. J. Path., 21: $1,1945$.

9. Lucas, A. M., and Riser, W. H. Intranuclear inclusions in panleukopenia of cats. A correlation with the pathogenesis of the disease and comparison with inclusions of herpes, B-virus, yellow fever, and burns. Am. J. Path., 21: 435, 1945.

10. Lillie, R. D., and Armstrong, Charles. Pathology of lymphocytic choriomeningitis in mice. Arch. Path., 40: 141, 1945.

11. Saphir, Otto. Visceral lesions in poliomyelitis. Am. J. Path., 21: 99, 1945.

12. Haythorn, S. R., and Taylor, F. A. Experimental silicosis produced with the ash from human silicotic lungs. Am. J. Path., 21: 123, 1945.

13. Gardner, L. U., and Heslington, H. F. Osteo-sarcoma from intravenous beryllium compounds in rabbits. Federation Proc., 5: 221, 1946.

14. Hirsch, E. F., and Russell, H. B. Chronic exudative and indurative pneumonia due to inhalation of shellac. Arch. Path., 39: 281, 1945.

15. Black-Schaffer, B. Pathology of anaphylaxis due to sulfonamide drugs. Arch. Path., 39: 301,1945 .

16. Herbut, P. A., and Scaricaciottoli, T. M. Diffuse hepatic necrosis caused by sulfadiazine. Arch. Path., 40: 94, 1945.

17. Moritz, A. R., Henriques, F. C., Jr., and McLean, Regina. The effects of inhaled heat on the air passages and lungs. An experimental investigation. Am. J. Path., 21: 311, 1945.

18. Baker, R. D. The internal lesions in burns with special reference to the liver and to splenic nodules. An analysis of 96 autopsies. Am. J. Path., 21: 717, 1945.

19. Gillman, J., and Gillman, T. Structure of the liver in pellagra. Arch. Path., 40: 239, 1945.

20. Wachstein, Max. Influence of dietary deficiencies and various poisons on the histochemical distribution of phosphatase in the liver. Arch. Path., 40: 57, 1945.

21. Fitzgerald, P. J., and Kinney, T. D. Intestinal lipodystrophy (Whipple's disease). Am. J. Path., 21: 1069, 1945.

22. Lindsay, S., and Knorp, W. F. Primary systemic amyloidosis. Arch. Path., 39: 315, 1945.

23. Pollak, O. J. Attempts to produce cerebral atherosclerosis. Arch. Path., 39: 16, 1945.

24. Dick, G. F. Experimental hypertension. Its production in dogs by intravenous injection of streptococci. Arch. Path., 39: 81, 1945.

25. Flory, C. M. Arterial occlusions produced by emboli from eroded atheromatous plaques. Am. J. Path., 21: 549, 1945.

26. Holyoke, J. B. Coronary arteriosclerosis and myocardial infarction as studied by an injection technic. Arch. Path., 39: 268, 1945.

27. Friedman, N. B. The pathology of trench foot. Am. J. Path., 21: 387, 1945.

28. Bevans, Margaret. Pathology of scleroderma, with special reference to the changes in the gastro-intestinal tract. Am. J. Path., 21: 25, 1945.

29. Bayley, E. C., Lindberg, D. O. N., and Baggenstoss, A. H. Loeffler's syndrome. Report of a case with pathologic examination of the lungs. Arch. Path., 40: 376, 1945. 